\title{
Universiteit
}

Leiden

The Netherlands

\section{Exact quantization of a paraxial electromagnetic field}

Aiello, A.; Woerdman, J.P.

\section{Citation}

Aiello, A., \& Woerdman, J. P. (2005). Exact quantization of a paraxial electromagnetic field.

Physical Review A, 72, 060101. doi:10.1103/PhysRevA.72.060101

Version: $\quad$ Not Applicable (or Unknown)

License: $\quad$ Leiden University Non-exclusive license

Downloaded from: https://hdl.handle.net/1887/61318

Note: To cite this publication please use the final published version (if applicable). 


\title{
Exact quantization of a paraxial electromagnetic field
}

\author{
A. Aiello and J. P. Woerdman \\ Huygens Laboratory, Leiden University, P.O. Box 9504, 2300 RA Leiden, The Netherlands
}

(Received 3 March 2005; published 30 December 2005)

\begin{abstract}
A nonperturbative quantization of a paraxial electromagnetic field is achieved via a generalized dispersion relation imposed on the longitudinal and the transverse components of the photon wave vector. This theoretical formalism yields a seamless transition between the paraxial- and the Maxwell-equation solutions. This obviates the need to introduce either $a d$ hoc or perturbatively defined field operators. Moreover, our (exact) formalism remains valid beyond the quasimonochromatic paraxial limit.
\end{abstract}

DOI: 10.1103/PhysRevA.72.060101

PACS number(s): 03.65.Db, 41.85.-p, 03.70.+k

In this Rapid Communication we seek an answer to the question: What is the quantum-mechanical state of a photon in a beam of light whose propagation can be classically described by a paraxial wave equation? The problem of quantum propagation of paraxial fields was considered by several authors in the past [1]. In these contributions the approach was either ad hoc or approximated. A notable exception was the work of Deutsch and Garrison where the authors developed a perturbative quantization scheme [2]. However, although their theory formally solves, order by order in a perturbation expansion, the problem of paraxial quantum propagation, it suffers from two main limitations. First, it does not provide any clear and easily manageable formula for the paraxial quantum modes. Second, it requires the quasimonochromatic approximation which is unsuitable for, e.g., the description of the very quantum phenomenon of propagation of broad-band entangled photons provided by spontaneous parametric down-conversion (SPDC). The increasing importance, for quantum information in general [3] and quantum cryptography [4] in particular, of a proper description of paraxial propagation of SPDC entangled photons, calls for an exact (namely, nonperturbative) theory of paraxial quantum fields.

The aim of this Rapid Communication is to introduce a nonperturbative method to quantize a paraxial electromagnetic field. Our scheme is conceptually simple: we begin by considering the quantized transverse electromagnetic vector potential calculated at the (arbitrary) initial time $t=0$. Then we select from the wave-vector space only those field configurations which are exact solutions of the paraxial wave equation, and let them evolve in time according to the d'Alembert wave equation. This procedure automatically ensures the validity of both the canonical commutation relations and the transversality conditions for the fields. We stress that our theory accounts for the axial propagation of vector fields with any spectral and spatial bandwidth and reproduces the well-known paraxial results in the limit of narrow beamlike fields.

To begin with, let us consider the vector potential operator $\hat{\mathbf{A}}(\mathbf{r}, t)=\hat{\mathbf{A}}^{(+)}(\mathbf{r}, t)+\hat{\mathbf{A}}^{(-)}(\mathbf{r}, t)$ in the Coulomb gauge which can be written in the plane-wave basis as [5]

$$
\begin{aligned}
\hat{\mathbf{A}}^{(+)}(\mathbf{r}, t)= & \int d^{3} \mathbf{k}\left(\frac{\hbar}{16 \pi^{3} \varepsilon_{0} c|\mathbf{k}|}\right)^{1 / 2} \\
& \times \sum_{\lambda=1}^{2} \boldsymbol{\epsilon}^{(\lambda)}(\mathbf{k}) \hat{a}_{\lambda}(\mathbf{k}) \exp (i \mathbf{k} \cdot \mathbf{r}-i c|\mathbf{k}| t),
\end{aligned}
$$

and $\hat{\mathbf{A}}^{(-)}(\mathbf{r}, t)$ is the Hermitian conjugate of $\hat{\mathbf{A}}^{(+)}(\mathbf{r}, t)$. It clearly satisfies the d' Alembert wave equation $\square \hat{\mathbf{A}}(\mathbf{r}, t)=0$. The two unit polarization vectors $\boldsymbol{\epsilon}^{(\lambda)}(\mathbf{k})(\lambda=1,2)$ are transverse $\boldsymbol{\epsilon}^{(\lambda)}(\mathbf{k}) \cdot \mathbf{k}=0$ and mutually orthogonal $\boldsymbol{\epsilon}^{(1)}(\mathbf{k}) \cdot \boldsymbol{\epsilon}^{(2)}(\mathbf{k})$ $=0$. Moreover, the annihilation and creation operators satisfy the canonical commutation rules $\left[\hat{a}_{\lambda}(\mathbf{k}), \hat{a}_{\lambda^{\prime}}^{\dagger}\left(\mathbf{k}^{\prime}\right)\right]$ $=\delta_{\lambda \lambda^{\prime}} \delta^{(3)}\left(\mathbf{k}-\mathbf{k}^{\prime}\right)$. Since the fields considered in this paper are mainly beams which propagate close to the $z$ direction, we find it convenient to introduce a finite quantization length $L$ along the $z$ axis with discrete wave vector Cartesian $z$ components $k_{z} \rightarrow \zeta_{n}=(2 \pi / L) n(n=0,1, \ldots)$. The choice $n \geqslant 0$ implies that only the parts of the field which propagate in the positive $z$ direction are included in Eq. (1). The integral with respect to $d k_{z}$ in Eq. (1) is then replaced by a sum[6,7] $\int d k_{x} d k_{y} d k_{z} \rightarrow(2 \pi / L) \Sigma_{n} \int d^{2} \mathbf{k}_{T}$, where $\quad \mathbf{k}_{T}=\left(k_{x}, k_{y}\right) \quad$ and $d^{2} \mathbf{k}_{T} \equiv d k_{x} d k_{y}$. Moreover, we scale the annihilation and creation operators by defining $\hat{a}_{\lambda}(\mathbf{k})=\sqrt{L /(2 \pi)} \hat{a}_{\lambda}\left(\mathbf{k}_{T}, n\right)$, in such a way that

$$
\left[\hat{a}_{\lambda}\left(\mathbf{k}_{T}, n\right), \hat{a}_{\lambda^{\prime}}^{\dagger}\left(\mathbf{k}_{T}^{\prime}, n^{\prime}\right)\right]=\delta_{\lambda \lambda^{\prime}} \delta_{n n^{\prime}} \delta^{(2)}\left(\mathbf{k}_{T}-\mathbf{k}_{T}^{\prime}\right)
$$

where the Kronecker symbol replaces the delta function according to $\delta\left(k_{z}-k_{z}^{\prime}\right) \rightarrow(L / 2 \pi) \delta_{n n^{\prime}}$.

A paraxial field is usually expressed as an envelope field modulating a carrier plane wave with wave vector $\mathbf{k}_{0}$ and angular frequency $\omega_{0}=c\left|\mathbf{k}_{0}\right|$. Without lack of generality we assume $\mathbf{k}_{0}=k_{0} \hat{\mathbf{z}}$ and we choose $k_{0}>0$ so that the carrier plane wave propagates in the positive $z$ direction. By using the trivial identity

$$
1=\frac{\mathcal{L}}{2 \pi} \int_{0}^{2 \pi / \mathcal{L}} d k_{0} \frac{\exp \left(i k_{0} z-j \omega_{0} t\right)}{\exp \left(i k_{0} z-i \omega_{0} t\right)},
$$

where $\mathcal{L}$ is an arbitrary length, we can rewrite the vector potential operator as 


$$
\begin{aligned}
\hat{\mathbf{A}}^{(+)}(\mathbf{r}, t) & =\hat{\mathbf{A}}^{(+)}(\mathbf{r}, t) \times 1 \\
& =\frac{\mathcal{L}}{2 \pi} \int_{0}^{2 \pi / \mathcal{L}} d k_{0} \exp \left(i k_{0} z-i \omega_{0} t\right) \hat{\mathbf{\Psi}}(\mathbf{r}, t),
\end{aligned}
$$

where we have introduced the envelope field

$$
\begin{aligned}
\hat{\mathbf{\Psi}}(\mathbf{r}, t)= & \sum_{n} \int d^{2} \mathbf{k}_{T}\left(\frac{\hbar}{8 \pi^{2} \varepsilon_{0} c|\mathbf{k}| L}\right)^{1 / 2} \sum_{\lambda=1,2} \boldsymbol{\epsilon}^{(\lambda)}(\mathbf{k}) \hat{a}_{\lambda}\left(\mathbf{k}_{T}, n\right) \\
& \times \exp \left[i\left(\mathbf{k}-k_{0} \hat{\mathbf{z}}\right) \cdot \mathbf{r}-i\left(c|\mathbf{k}|-\omega_{0}\right) t\right],
\end{aligned}
$$

where $\mathbf{k}=\mathbf{k}_{T}+\hat{\mathbf{z}} \zeta_{n}$. Now, the key idea is to find a subspace of the three-dimensional wave vector $\mathbf{k}$ space where the initial time envelope field $\hat{\boldsymbol{\Psi}}(\mathbf{r}) \equiv \hat{\boldsymbol{\Psi}}(\mathbf{r}, t=0)$ satisfies the paraxial wave equation [8]

$$
\frac{\partial^{2} \hat{\boldsymbol{\Psi}}(\mathbf{r})}{\partial x^{2}}+\frac{\partial^{2} \hat{\boldsymbol{\Psi}}(\mathbf{r})}{\partial y^{2}}+2 i k_{0} \frac{\partial \hat{\mathbf{\Psi}}(\mathbf{r})}{\partial z}=0 .
$$

If we substitute from Eq. (5) into Eq. (6) we obtain

$$
\zeta_{n} / k_{0}=1-\left|\mathbf{k}_{T}\right|^{2} /\left(2 k_{0}^{2}\right) .
$$

This generalized dispersion relation plays a key role through this paper. It defines a two-dimensional domain in the $\mathbf{k}$ space where both d'Alembert wave equation and paraxial wave equation are satisfied. For sake of clarity we write $\mathbf{q}=\mathbf{k}_{T}, \quad q=|\mathbf{q}|$ and define the dimensionless parameter $\vartheta=q /\left(\sqrt{2 k_{0}^{2}}\right)$. If we denote with $\theta \in[0, \pi / 2]$ the angle between the wave vector $\mathbf{k}$ and the axis $z$, then $q / \mathbf{k} \cdot \hat{\mathbf{z}}=\tan \theta$ and from Eq. (7), it follows that $\vartheta \sqrt{2}=-\cot \theta+\sqrt{\left(2+\cot ^{2} \theta\right)}$. This relation is exact; however, we can gain some insight if we consider it in the limit $\theta \ll 1$ where $\vartheta \sqrt{2} \simeq \theta-\theta^{3} / 6+O\left(\theta^{5}\right)$. This equations shows that $\vartheta \sqrt{2}$ $=q / k_{0}$ is approximately equal to the divergence angle of a Gaussian beam[9]. Moreover, by comparing $\vartheta$ with Eq. (2.8) by Deutsch and Garrison[2], one recognizes $\vartheta \sqrt{2} \ll 1$ as their perturbative expansion parameter. However, in our case the only constraint is $\vartheta \leqslant 1$, as follows from Eq. (7) and the condition $\zeta_{n} \geqslant 0$.

From Eq. (7) it readily follows that the exponential factor in Eq. (5) can be written

$$
\begin{aligned}
\exp & {\left[i\left(\mathbf{k}-k_{0} \hat{\mathbf{z}}\right) \cdot \mathbf{r}-i\left(c|\mathbf{k}|-\omega_{0}\right) t\right] } \\
\quad & \exp \left(i \mathbf{q} \cdot \mathbf{x}-i \vartheta^{2} k_{0} z\right) \exp \left[-i \omega_{0} t\left(\sqrt{1+\vartheta^{4}}-1\right)\right],
\end{aligned}
$$

where $\mathbf{x} \equiv(x, y)$. Clearly Eq. (7) affects also the value of the polarization unit vectors in Eq. (5). To see this, we first write the total wave vector $\mathbf{k}$ in terms of $\mathbf{q}$ and $k_{0}$ as $\mathbf{k}=\hat{\mathbf{q}} q+\hat{\mathbf{z}} k_{0}\left(1-\vartheta^{2}\right)$, where $\hat{\mathbf{q}} \equiv \mathbf{q} /|\mathbf{q}|$, then we arbitrarily choose (we always have this freedom) $\boldsymbol{\epsilon}^{(2)}(\mathbf{q}, \vartheta)=\hat{\mathbf{z}} \times \hat{\mathbf{q}}$. The remaining unit vector $\boldsymbol{\epsilon}^{(1)}$ is then uniquely fixed by the cyclic relation $\boldsymbol{\epsilon}^{(1)} \propto \boldsymbol{\epsilon}^{(2)} \times \mathbf{k}$ to the value

$$
\boldsymbol{\epsilon}^{(1)}(\mathbf{q}, \vartheta)=\left[\hat{\mathbf{q}}\left(1-\vartheta^{2}\right)-\hat{\mathbf{z}} \vartheta \sqrt{2}\right] /\left(1+\vartheta^{4}\right)^{1 / 2} .
$$

It is now possible to write explicitly the envelope field $\hat{\boldsymbol{\Psi}}(\mathbf{r})$ restricted to the $\mathbf{k}$ subspace defined by the dispersion relation Eq. (7). From the definition of $\zeta_{n}$ and Eq. (7), it follows that we must select from the sum over $n$ in Eq. (5) only those terms corresponding to $n=n(\vartheta) \equiv\left[\left(k_{0} L / 2 \pi\right)\left(1-\vartheta^{2}\right)\right]_{\mathrm{IP}}$, where "IP" stands for integer part. This objective can be achieved by replacing in Eq. (5) $\Sigma_{n} \rightarrow \Sigma_{n} \delta_{n, n(\vartheta)}$, where $\delta_{n, n(\vartheta)}=1$ for $n=n(\vartheta)$ and $\delta_{n, n(\vartheta)}=0$ otherwise. Finally, we can write from Eq. (5)

$$
\begin{aligned}
\hat{\boldsymbol{\Psi}}(\mathbf{r})= & \sum_{n} \int d^{2} \mathbf{q}\left(\frac{\hbar}{8 \pi^{2} \varepsilon_{0} \omega_{0}\left(1+\vartheta^{4}\right)^{1 / 2} L}\right)^{1 / 2} \\
& \times \sum_{\lambda=1,2} \boldsymbol{\epsilon}^{(1)}(\mathbf{q}, \vartheta) \hat{a}_{\lambda}(\mathbf{q}, n) \delta_{n, n(\vartheta)} \\
& \times \exp \left(i \mathbf{q} \cdot \mathbf{x}-i \vartheta^{2} k_{0} z\right),
\end{aligned}
$$

where $\boldsymbol{\epsilon}^{(\lambda)}(\mathbf{q}, \vartheta)$ are given by Eq. (9) and previous formulas. We have substituted everyplace in Eq. (10) $\zeta_{n}$ by Eq. (7); this operation is permitted by the presence of $\delta_{n, n(\vartheta)}$ within the sum. The only exception to these substitutions is represented by the operators $\hat{a}_{\lambda}(\mathbf{q}, n)$ which, for the moment, are left unchanged.

At this point, we note that since the restriction to the paraxial $\mathbf{k}$ subspace has already been achieved via Eq. (10), it is possible to make a step backward from the discrete momentum $\zeta_{n}$ to the continuous paraxial frequency $\omega: c \zeta_{n}$ $=(2 \pi c / L) n \rightarrow \omega$. The reason for this choice for the name of $\omega$, will become clear later [see discussion after Eq. (23)]. The other required replacements are $\Sigma_{n} \rightarrow(L / 2 \pi c) \int d \omega$, $\hat{a}_{\lambda}(\mathbf{q}, n)=\sqrt{2 \pi c / L} \hat{a}_{\lambda}(\mathbf{q}, \omega)$ and

$$
\delta_{n, n(\vartheta)}=\frac{2 \pi c}{L} \delta\left(\omega-c k_{0}\left(1-\vartheta^{2}\right)\right)
$$

in such a way that

$$
\left[\hat{a}_{\lambda}(\mathbf{q}, \omega), \hat{a}_{\lambda^{\prime}}^{\dagger}\left(\mathbf{q}^{\prime}, \omega^{\prime}\right)\right]=\delta_{\lambda \lambda^{\prime}} \delta\left(\omega-\omega^{\prime}\right) \delta^{(2)}\left(\mathbf{q}-\mathbf{q}^{\prime}\right) .
$$

Equation (4) can be then written as

$$
\begin{aligned}
\hat{\mathbf{A}}^{(+)}(\mathbf{r}, t)= & \left(\frac{\mathcal{L}}{L}\right) \int_{0}^{2 \pi / \mathcal{L}} d k_{0} \exp \left(i k_{0} z-i \omega_{0} t\right) \int d \omega \int d^{2} \mathbf{q} \\
& \times\left(\frac{\hbar}{16 \pi^{3} \varepsilon_{0} k_{0}\left(1+\vartheta^{4}\right)^{1 / 2}}\right)^{1 / 2} \\
& \times \sum_{\lambda=1,2} \boldsymbol{\epsilon}^{(\lambda)}(\mathbf{q}, \vartheta) \hat{a}_{\lambda}(\mathbf{q}, \omega) \delta\left(\omega-c k_{0}\left(1-\vartheta^{2}\right)\right) \\
& \times \exp \left(i \mathbf{q} \cdot \mathbf{x}-i \vartheta^{2} k_{0} z\right) \exp \left[-i \omega_{0} t\left(\sqrt{1+\vartheta^{4}}-1\right)\right],
\end{aligned}
$$

where the irrelevant term $\mathcal{L} / L$ can be absorbed in the definition of $\hat{\mathbf{A}}^{(+)}(\mathbf{r}, t)$. This is not yet our final expression since we can perform explicitly the integration with respect to $\omega$ which is still present in the expression for the annihilation operator:

$$
\int d \omega \hat{a}_{\lambda}(\mathbf{q}, \omega) \delta\left(\omega-c k_{0}\left(1-\vartheta^{2}\right)\right) \equiv \sqrt{1+\vartheta^{2}} \hat{a}_{\lambda}\left(\mathbf{q}, \omega_{0}\right),
$$

where 


$$
\left[\hat{a}_{\lambda}\left(\mathbf{q}, \omega_{0}\right), \hat{a}_{\lambda^{\prime}}^{\dagger},\left(\mathbf{q}^{\prime}, \omega_{0}^{\prime}\right)\right]=\delta_{\lambda^{\prime}}, \delta^{(2)}\left(\mathbf{q}-\mathbf{q}^{\prime}\right) \delta\left(\omega_{0}-\omega_{0}^{\prime}\right)
$$

The use of Eq. (14) has been suggested to us by Visser and Nienhuis who first introduced this technique [11]. Finally, we can write, after redefining $c k_{0} \equiv \omega$,

$$
\begin{aligned}
\hat{\mathbf{A}}^{(+)}(\mathbf{r}, t)= & \int d \omega \exp [-i \omega(t-z / c)]\left(\frac{\hbar}{16 \pi^{3} \varepsilon_{0} c \omega}\right)^{1 / 2} \int d^{2} \mathbf{q} \\
& \times \sum_{\lambda=1,2} \mathcal{E}^{(\lambda)}(\mathbf{q}, \omega, z, t) \hat{a}_{\lambda}(\mathbf{q}, \omega) \exp \left(i \mathbf{q} \cdot \mathbf{x}-i \frac{q^{2} c}{2 \omega} z\right),
\end{aligned}
$$

where we have defined the slowly varying polarization vectors

$$
\begin{aligned}
\mathcal{E}^{(\lambda)}(\mathbf{q}, \omega, z, t) \equiv & \boldsymbol{\epsilon}^{(\lambda)}(\mathbf{q}, \vartheta)\left(\frac{1+\vartheta^{2}}{\sqrt{1+\vartheta^{4}}}\right)^{1 / 2} \\
& \times \exp \left[-i \omega t\left(\sqrt{1+\vartheta^{4}}-1\right)\right],
\end{aligned}
$$

and the unit vectors $\boldsymbol{\epsilon}^{(\lambda)}(\mathbf{q}, \vartheta)$ are given by Eq. (9) and previous formulas.

Equation (16) is the first main result of this work. It represents a vector potential field operator which is a bona fide transverse field obeying the d'Alembert wave equation for any time $t>0$, whose corresponding envelope field satisfies the paraxial wave equation (6) at $t=0$. We stress that this expression is exact; no approximations were made.

Now, we are ready to address the problem of building the quantum mechanical state describing a photon in a paraxial beam. To this end, we first note that until now the creation operators $\hat{a}_{\lambda}^{\dagger}(\mathbf{q}, \omega)$ have passed untouched through all our operations. In fact, they still satisfy the canonical commutation relations Eq. (12). However, their form is not the most suitable one to deal with paraxial fields; therefore we introduce the Fourier-transformed operators[7,10]

$$
\hat{a}_{\lambda}(\mathbf{x}, \omega)=\frac{1}{2 \pi} \int d^{2} \mathbf{q} \hat{a}_{\lambda}(\mathbf{q}, \omega) \exp (i \mathbf{q} \cdot \mathbf{x})
$$

such that

$$
\left[\hat{a}_{\lambda}(\mathbf{x}, \omega), \hat{a}_{\lambda^{\prime}}^{\dagger}\left(\mathbf{x}^{\prime}, \omega^{\prime}\right)\right]=\delta_{\lambda \lambda^{\prime}} \delta\left(\omega-\omega^{\prime}\right) \delta^{(2)}\left(\mathbf{x}-\mathbf{x}^{\prime}\right)
$$

If we substitute Eq. (18) in Eq. (16) we obtain, after some algebra,

$$
\hat{\mathbf{A}}^{(+)}(\mathbf{r}, t)=\sum_{\lambda=1}^{2} \int d \omega \frac{e^{-i \omega(t-z / c)}}{\left(4 \pi \varepsilon_{0} c \omega / \hbar\right)^{1 / 2}} \hat{\mathcal{A}}^{(\lambda)}(\mathbf{x}, z, \omega, t),
$$

where $\mathbf{r}=(\mathbf{x}, z)$ and we have introduced the exact slowly varying photon annihilation vector operators

$$
\hat{\mathcal{A}}^{(\lambda)}(\mathbf{x}, z, \omega, t) \equiv \int d^{2} \mathbf{x}^{\prime} \mathcal{F}^{(\lambda)}\left(\mathbf{x}, z, \mathbf{x}^{\prime}, \omega, t\right) \hat{a}_{\lambda}\left(\mathbf{x}^{\prime}, \omega\right)
$$

Moreover, in Eq. (21) we have defined the Maxwell-paraxial (MP) slowly varying modes

$$
\begin{aligned}
\mathcal{F}^{(\lambda)}\left(\mathbf{x}, z, \mathbf{x}^{\prime}, \omega, t\right)= & \frac{1}{(2 \pi)^{2}} \int d^{2} \mathbf{q} \mathcal{E}^{(\lambda)}(\mathbf{q}, \omega, z, t) \\
& \times \exp \left[i \mathbf{q} \cdot\left(\mathbf{x}-\mathbf{x}^{\prime}\right)-i \frac{q^{2} c}{2 \omega} z\right] .
\end{aligned}
$$

Equation (22) displays the second main result of this paper. It describes the field in the plane $z$ at time $t$ due to a point source with paraxial frequency $\omega$ located at $\mathbf{x}^{\prime}$ in the transverse plane $z=0$. As its shape clearly suggests, $\mathcal{F}^{(\lambda)}\left(\mathbf{x}, z, \mathbf{x}^{\prime}, \omega, t\right)$ is the quantum analog of the classical Huygens-Fresnel diffracted field. This may be seen more clearly by writing Eq. (22) in the narrow-beam limit which is achieved by restricting the transverse momentum integral to the domain $\mathcal{C}_{\omega}=\{\mathbf{q}: q \ll \omega / c\}$. It is easy to see that within this domain $\vartheta=q c / \omega \sqrt{2} \ll 1$, and $\mathcal{E}^{(\lambda)}(\mathbf{q}, \omega, z, t) \simeq \mathbf{e}^{(\lambda)}(\mathbf{q})$, where $\mathbf{e}^{(1)}(\mathbf{q})=\hat{\mathbf{q}}+O(\vartheta)$ and $\mathbf{e}^{(2)}(\mathbf{q})=\hat{\mathbf{z}} \times \hat{\mathbf{q}}$, are the zeroth order polarization unit vectors. In this limit Eq. (22) reduces to the well-known paraxial Green's function $\mathcal{P}^{(\lambda)}\left(\mathbf{x}, z, \mathbf{x}^{\prime}, \omega\right)[8]$

$$
\mathcal{P}^{(\lambda)}\left(\mathbf{x}, z, \mathbf{x}^{\prime}, \omega\right)=\int_{\mathcal{C}_{\omega}} \frac{d^{2} \mathbf{q}}{(2 \pi)^{2}} \mathbf{e}^{(\lambda)}(\mathbf{q}) e^{i\left[\mathbf{q} \cdot\left(\mathbf{x}-\mathbf{x}^{\prime}\right)-\left(q^{2} c / 2 \omega\right) z\right]},
$$

here generalized to vector fields. From Eq. (23) it is clear that in the narrow-beam limit the paraxial frequency reduces to the actual frequency $\omega$ and $\hat{\mathcal{A}}^{(\lambda)}(\mathbf{x}, z, \omega, t)$ becomes time independent. Now, it is straightforward to show that at a fixed time $t$, in each transverse plane $z$, the MP functions $\mathcal{F}^{(\lambda)}\left(\mathbf{x}, z, \mathbf{x}^{\prime}, \omega, t\right)$ are quasiorthogonal

$$
\begin{aligned}
& \int d^{2} \mathbf{x} \mathcal{F}^{(\lambda)}\left(\mathbf{x}, z, \mathbf{x}^{\prime}, \omega, t\right) \cdot \mathcal{F}^{(\mu)}\left(\mathbf{x}, z, \mathbf{x}^{\prime \prime}, \omega, t\right) \\
& =\delta_{\lambda \mu} \int \frac{d^{2} \mathbf{q}}{(2 \pi)^{2}}\left(\frac{1+\vartheta^{2}}{\sqrt{1+\vartheta^{4}}}\right)^{1 / 2} \exp \left[i \mathbf{q} \cdot\left(\mathbf{x}^{\prime}-\mathbf{x}^{\prime \prime}\right)\right],
\end{aligned}
$$

that is, the right side of this equation approaches $\delta_{\lambda \mu} \delta^{(2)}\left(\mathbf{x}^{\prime}-\mathbf{x}^{\prime \prime}\right)$ in the narrow-beam limit $\vartheta \rightarrow 0$, as expected from the orthogonality of the classical paraxial Green's functions for free space propagation. This result was already found by Visser and Nienhuis[11] who suggested to interpret the Fourier-transformed creation operator $\hat{a}_{\lambda}^{\dagger}(\mathbf{x}, \omega)$ as the operator which creates at $t=0$ a photon with polarization $\lambda$ in the paraxial mode $\exp (i \omega z / c) \mathcal{P}^{(\lambda)}\left(\mathbf{x}, z, \mathbf{x}^{\prime}, \omega\right)$. More generally, the interpretation of $\mathcal{F}^{(\lambda)}\left(\mathbf{x}, z, \mathbf{x}^{\prime}, \omega, t\right)$ as single-photon wave function can be put on a rigorous basis by introducing the "transverse-position" states $|\mathbf{x}, \omega, \lambda\rangle \equiv \hat{a}_{\lambda}^{\dagger}(\mathbf{x}, \omega)|0\rangle$. Then it readily follows that $\left\langle 0\left|\hat{\mathbf{A}}^{(+)}\left(\mathbf{x}^{\prime}, z, t\right)\right| \mathbf{x}, \omega, \lambda\right\rangle$ $\propto \mathcal{F}^{(\lambda)}\left(\mathbf{x}^{\prime}, z, \mathbf{x}, \omega, t\right) \exp [-i \omega(t-z / c)]$.

Now, the definition of $|\mathbf{x}, \omega, \lambda\rangle$ makes it possible to asso- 
ciate to any single-photon state $|\psi\rangle$, its corresponding Maxwell-paraxial wave function $\psi_{\lambda}(\mathbf{x}, \omega) \equiv\langle\mathbf{x}, \omega, \lambda \mid \psi\rangle$. Then, for example, the MP wave function associated to the plane wave state $|\mathbf{q}, \omega, \lambda\rangle \equiv \hat{a}_{\lambda}^{\dagger}(\mathbf{q}, \omega)|0\rangle$, is simply given by the Fourier relation $\left\langle\mathbf{x}, \omega^{\prime}, \lambda^{\prime} \mid \mathbf{q}, \omega, \lambda\right\rangle$ $=[\exp (i \mathbf{q} \cdot \mathbf{x}) / 2 \pi] \delta_{\lambda^{\prime}} \delta\left(\omega-\omega^{\prime}\right)$. More generally, for a given complete set of orthogonal transverse functions $\psi_{n m}(\mathbf{x}, \omega)$ as, e.g., the Hermite- or the Laguerre-Gaussian beams [9], it is possible to build the corresponding MP single-photon state as

$$
|n, m, \omega, \lambda\rangle=\int d^{2} \mathbf{x} \psi_{n m}(\mathbf{x}, \omega)|\mathbf{x}, \omega, \lambda\rangle,
$$

where $\left\langle\mathbf{x}, \omega^{\prime}, \lambda^{\prime} \mid n, m, \omega, \lambda\right\rangle=\psi_{n m}(\mathbf{x}, \omega) \delta_{\lambda \lambda^{\prime}} \delta\left(\omega-\omega^{\prime}\right)$.

This equation is our third and final main result: it represents the exact quantum-mechanical state of each of the photons in the classical paraxial beam $\psi_{n m}(\mathbf{x}, \omega)$. It should be noticed that while Eq. (25) is always well defined in our perfectly general framework, its interpretation in terms of paraxial beams is limited by the classical condition $\tilde{\psi}_{n m}(\mathbf{q}, \omega) \approx 0$ for $q \gtrsim \omega / c$, where $\tilde{\psi}_{n m}(\mathbf{q}, \omega)$ is the twodimensional Fourier transform of $\psi_{n m}(\mathbf{x}, \omega)$.

In conclusion, a nonperturbative quantization scheme for electromagnetic paraxial fields has been introduced. It relies on the fact that it is possible to select some initial field configurations which are exact solutions of the paraxial wave equation. These configurations are then evolved at later times with the d'Alembert wave equation. In this way we were able to find explicit and manageable expressions for the exact field in both momentum [Eq. (16)] and in position [Eqs. (20)-(22)] representation. Moreover we gave an unambiguous definition for Maxwell-paraxial quantum states [Eq. (25)]. The usefulness of the present theory, first introduced by us in [12], has been very recently confirmed by Calvo et al. [13], who used our formalism to illustrate some properties of photon angular momentum.

It is a pleasure to acknowledge Jorrit Visser and Gerard Nienhuis for fruitful discussions. We also acknowledge support from the EU under the IST-ATESIT contract. This project was also supported by FOM.
[1] R. Graham and H. Haken, Z. Phys. 213, 420 (1968); H. Yuen and J. H. Shapiro, IEEE Trans. Inf. Theory IT-24, 657 (1978); T. A. B. Kennedy and E. M. Wright, Phys. Rev. A 38, 212 (1988); J. C. Garrison, H. Nathel, and R. Y. Chiao, J. Opt. Soc. Am. B 5, 1528 (1988); M. I. Kolobov, Rev. Mod. Phys. 71, 1539 (1999).

[2] I. H. Deutsch and J. C. Garrison, Phys. Rev. A 43, 2498 (1991).

[3] M. A. Nielsen and I. L. Chuang, Quantum Computation and Quantum Information, reprinted 1st ed. (Cambridge University Press, Cambridge, UK, 2002).

[4] N. Gisin, G. Ribody, W. Tittel, and H. Zbinden, Rev. Mod. Phys. 74, 145 (2002).

[5] R. Loudon, The Quantum Theory of Light, 3rd ed. (Oxford University Press, Oxford, UK, 2000).

[6] T. D. Lee, Particle Physics and Introduction to Field Theory, revised and updated 1st ed. (Harwood Academic, Chur, Switzerland, 1988).

[7] K. J. Blow, R. Loudon, S. J. D. Phoenix, and T. J. Shepherd, Phys. Rev. A 42, 4102 (1990).

[8] L. Mandel and E. Wolf, Optical Coherence and Quantum Optics 1st ed. (Cambridge University Press, Cambridge, 1995).

[9] A. E. Siegman, Lasers (University Science Books, Mill Valley, CA, 1996).

[10] A. F. Abouraddy, B. E. A. Saleh, A. V. Sergienko, and M. C. Teich, Phys. Rev. Lett. 87, 123602 (2001).

[11] G. Nienhuis (private communication); J. Visser, Ph.D. thesis, Leiden University, 2005, (unpublished).

[12] A. Aiello and J. P. Woerdman, e-print quant-ph/0502164.

[13] G. F. Calvo, A. Picón, and E. Bagan, e-print quant-ph/ 0509040 . 